\title{
The expression of Axl receptor tyrosine kinase influences the tumour phenotype and clinical outcome of patients with malignant pleural mesothelioma
}

\author{
D J Pinato ${ }^{1,7}$, F A Mauri ${ }^{2,7}$, T Lloyd ${ }^{2}$, V Vaira ${ }^{3}$, C Casadio ${ }^{4}$, R L Boldorini ${ }^{5}$ and R Sharma ${ }^{*}, 1,6$
}

${ }^{1}$ Department of Experimental Medicine, Imperial Center for Translational and Experimental Medicine, Imperial College London, Du Cane Road, W12 OHS London, UK; ${ }^{2}$ Department of Histopathology, Imperial College London, Du Cane Road, W12 OHS London, UK; ${ }^{3}$ Division of Pathology, Fondazione IRCCS Ca' Granda Ospedale Maggiore Policlinico, via F. Sforza 35, 20122 Milan, Italy; ${ }^{4}$ Department of Health Sciences, Division of Thoracic Surgery, Università del Piemonte Orientale 'Amedeo Avogadro', 27100 Novara, Italy; ${ }^{5}$ Department of Health Sciences, Division of Histopathology, Università del Piemonte Orientale 'Amedeo Avogadro', 27100 Novara, Italy and 'Department of Medical Oncology, Imperial College London, Hammersmith Campus, Du Cane Road, W12 OHS London, UK

Background: Recent preclinical studies identified Axl, a tyrosine kinase receptor implicated in tumour progression and epithelialto-mesenchymal transition, as a putative therapeutic target in malignant pleural mesothelioma (MPM), an invariably fatal malignancy with limited treatment options. Here, we studied the expression of Axl and its ligand Gas-6 (growth arrest signal-6) in primary specimens of MPM, correlating their expression levels with tumour phenotype and clinical outcomes.

Methods: Two independent cohorts of consecutive patients diagnosed with MPM were studied: a derivation cohort composed of 63 cases and a validation set of 35 cases. Clinical variables including patients' demographics, tumour stage, histotype, performance status (PS), Axl and Gas-6 staining were tested for predicting overall survival (OS) using univariate and multivariate analyses.

Results: In the derivation cohort, $\mathrm{Axl}(P=0.001)$ but not Gas-6 overexpression $(P=0.35)$ emerged as a univariate prognostic factor for OS, together with stage $(P=0.05)$, PS $(P<0.001)$ hypoalbuminaemia $(P<0.001)$ and anaemia $(P<0.001)$. Multivariate analyses confirmed $A x l$ overexpression $(P=0.01)$, PS $(P=0.01)$, hypoalbuminaemia $(P<0.001)$ and anaemia $(P=0.04)$ as independent predictors of OS. The prognostic role of $A x l$ overexpression was externally validated in an independent cohort $(P=0.03)$.

Conclusion: Overexpression of Axl is found in the majority of MPM specimens and influences patient's survival independently from other established prognostic factors. Such information may support patient selection for future trials.

Malignant pleural mesothelioma (MPM) is an aggressive tumour with an invariably poor prognosis, whose incidence is expected to peak by 2020 in Western countries and to remain elevated in developing areas of the world as a result of the enduring marketing of asbestos (Joshi and Gupta, 2004). Radical treatment protocols often combine induction platinum-based chemotherapy and surgery followed by adjuvant radiotherapy to the involved hemithorax (Weder et al, 2007). However, this approach is only feasible in a carefully selected fraction of patients who present with MPM (Hasani et al, 2009) and recent evidence from retrospective

${ }^{*}$ Correspondence: Dr R Sharma; E-mail: r.sharma@imperial.ac.uk
${ }^{7}$ These authors contributed equally to this work.

Received 5 October 2012; revised 17 December 2012; accepted 18 December 2012; published online 29 January 2013

(C) 2013 Cancer Research UK. All rights reserved 0007 - 0920/13 
(Rena and Casadio, 2012) as well as prospective randomised studies (Treasure et al, 2011) has cast doubts upon its role in improving survival and quality of life. As the majority of patients are diagnosed with inoperable disease, palliative chemotherapy in the form of pemetrexed and cisplatin is the most practiced therapeutic strategy in MPM, yet with limited effects in achieving long-term overall survival (OS), rarely exceeding 12 months (Vogelzang et al, 2003).

In the past 20 years, increasing research efforts have been addressed to better characterise the molecular nature of this invariably fatal malignancy, with the overall aim of unveiling the molecular drivers of unrestrained tumour progression that confer both an adverse prognosis and resistance to conventional treatments(Greillier et al, 2008). Activation of receptor tyrosine kinases (RTK) and their downstream signalling pathways by either RTK mutation/amplification or by the superabundant secretion of growth factors acting through autocrine or paracrine loops has been recognised as a major molecular trait in the pathogenesis and the prognosis of MPM (Jean et al, 2012). Previous studies have shown that the overexpression of a subset of RTKs, including epidermal growth factor receptor (EGF-R) and platelet-derived growth factor receptor (PDGF-R), is typical of MPM (Arber et al, 2004; Destro et al, 2006) and correlates with prognosis. However, such knowledge has not translated into a significant improvement in the clinical management of the disease, with EGF-R or PDGF-R inhibitors lacking clinical efficacy in proof of concept studies (Mathy et al, 2005; Garland et al, 2007). Nevertheless, besides the described therapeutic implications, the prognostic significance observed for the activation of RTK-mediated signalling pathways lends themselves as an invaluable source of novel and objective predictors of survival outcomes, whose clinical role could be that of improving treatment allocation in MPM, reserving, for instance, radical approaches to those patients with less-aggressive disease and a reasonable likelihood of achieving long-term survival.

Recently published work has identified the oncogene Axl, a member of the TAM RTK subfamily, as a potential novel therapeutic target in MPM (Ou et al, 2011a). Identified as a transforming gene across a wide array of solid tumours, Axl shares the common ligand growth arrest signal-6 (Gas-6) with the other TAM RTKs Mer and Tyro-3. Upon ligation with Gas-6, Axl directly mediates the concurrent activation of the RAS/RAF/ MAPK (Hasanbasic et al, 2004) and the PI3K/AKT/S6K intracellular signalling cascades, which functionally converge in promoting tumour cell survival and evasion from apoptosis (Fridell et al, 1996). More recently, overexpression of Axl has also been illustrated to be a key step in epithelial-to-mesenchymal transition (EMT), through which cancer cells lose key adhesion molecules such as E-cadherin and acquire unrestrained cell motility and metastatic potential (Gjerdrum et al, 2010).

Despite mechanistic studies demonstrating that Axl inhibition can suppress mesothelioma proliferation and invasion in vitro (Ou et al, 2011a), further research is needed to understand how Axl expression can shape the clinical phenotype of MPM. Furthermore, if prospective clinical evaluation of Axl-targeted inhibition is warranted, evaluation of the prevalence of Axl expression in MPM is desirable to enable patient stratification for future studies. In the present study, we used a retrospective multicenter collection of primary tissue specimens to identify the prevalence of Axl and Gas-6 expression in MPM and to fully elucidate their prognostic role.

\section{MATERIALS AND METHODS}

Patient population. From a database of 171 patients diagnosed with MPM within Imperial College NHS Trust (Pinato et al, 2012), 63 consecutive cases with available archival tissue samples were included to produce a derivation set. All patients were diagnosed with MPM between 2001 and 2011. Histopathological diagnosis and histotype subclassification was confirmed in all cases following haematoxylin and eosin slides review by a board certified pathologist (FAM). Nine specimens (14\%) were surgical samples obtained following pleurectomy/decortication procedures, whereas the remaining $54(86 \%)$ were core biopsy samples. Complete clinicopathological data including demographics and staging information were collected along with the complete blood picture and performance status (PS) computed according to the Eastern Cooperative Oncology Group criteria. Radiological tumour staging at diagnosis followed the International Mesothelioma Interest Group (IMIG) criteria (Rusch, 1995). Patients were subgrouped in two categories (stages I-II and III-IV). The European Organisation for Research and Treatment of Cancer Prognostic Score (EPS) was derived as described previously and categorises patients into good $v s$ poor prognostic groups according to male gender, probable or possible histologic diagnosis, sarcomatoid differentiation, $\mathrm{PS} \geqslant 1$, and $\mathrm{WBC} \geqslant 8.3 \times 10^{9} 1^{-1}$ (Fennell et al, 2005). Overall (cancer specific) survival was calculated from the time of histological diagnosis to the time of patients' death or last follow-up. An independent series of 35 core biopsy samples of MPM diagnosed at the Maggiore della Carità University Hospital (Piemonte Orientale University, Novara, Italy) between 2005 and 2010 was selected to validate the immunohistochemistry analysis.

Immunohistochemistry. Expression of the candidate biomarkers was studied by immunohistochemistry using a modified version of the antigen retrieval protocol firstly described by Shi et al (1991). Briefly, tissue sections were de-paraffinised in xylene, rehydrated in graded alcohols and heated in a microwave oven at $900 \mathrm{~W}$ for $30 \mathrm{~min}$ in citrate buffer at $\mathrm{pH}$ 6.0. Before immunostaining, slides were cooled at room temperature and quenching of endogenous peroxidase activity was obtained by incubation with a $3 \%$ solution of $\mathrm{H}_{2} \mathrm{O}_{2}$ for $5 \mathrm{~min}$. The primary antibodies were incubated overnight at the concentration of 1:50 for anti Gas-6 (Cat. nr. HPA008275, Sigma Aldrich, St Louis, MO, USA), Axl (Cat. nr. HPA037422, Sigma Aldrich) and human telomerase reverse transcriptase (hTERT clone Y182, Novus Biologicals, Cambridge, UK) antisera. Immunostaining for Ki-67 (Leica Microsystems, Wetzlar, Germany) and vascular endothelial growth factor A (VEGF-A, Santa Cruz Biotechnology, Santa Cruz, CA, USA) was carried out at 1:800 and 1:750 dilution, respectively. Tissue sections were incubated with the secondary antibody for $1 \mathrm{~h}$ at room temperature and then processed using the Polymer-HRP Kit (BioGenex, San Ramon, CA, USA) with development in Diaminobenzidine and Mayer's Haematoxylin counterstaining. Appropriately chosen tissue samples were used as external positive control during each reaction to confirm its specificity. Omission of the primary antibody was used as negative control reactions, resulting in the absence of staining in all cases. All the immunostaining procedures were performed centrally within the Imperial College NHS Trust Pathology Department-Hammersmith Hospital.

Protein expression was quantified using the histoscore (HS) method. Briefly, each tumour specimen was scored on a semiquantitative scale ranging from 0 to 300 , with the final score resulting from the percentage of tumour cells staining positively (range 0-100) multiplied by staining intensity graded as negative, weak, moderate or strong (range $0-3$ ). The Ki-67 labelling index was expressed as the percentage of immunopositive nuclei from a minimum of 500 nuclei in at least five microscopic fields. The median HS value was used as a cutoff level to discriminate high vs low expression of each biomarker. Two observers (FAM, DJP) blinded to the clinical data scored all the cases and consensus was reached in case of significant discrepancy between the individual scores.

Immunostaining for VEGF-A and Ki-67 was carried out in a smaller subgroup of 20 specimens selected at random from the 
derivation cohort to study the relationship between Axl and Gas-6 expression and tumour angiogenesis and proliferative potential. Histological representation in the subgroup was comparable with the original series, with $75 \%$ of the tumours being of epithelioid histotype. Samples were categorised as having high vs low proliferation index or VEGF-A expression based on the median nuclear count (30) and VEGF-A HS value (90) of the distribution, respectively. A total of 10 cases $(50 \%)$ had a Ki-67 nuclear count $\geqslant 30$, whereas 9 (45\%) had VEGF-A HS values $\geqslant 90$. We also performed an ad hoc subanalysis on a further subgroup of 20 specimens selected from the derivation group based on high $(\mathrm{HS} \geqslant 150)$ vs low $(\mathrm{HS}<150)$ Axl expression levels to investigate whether Axl expression influenced tumour cell senescence.

Statistical analysis. Pearson's $\chi^{2}$ or Fisher's exact tests were used to evaluate any significant associations between categorical variables as appropriate. Associations were considered statistically significant when the resulting $P$-value was $<0.05$ (two sided). Kaplan-Meier statistics and Log Rank test were used to study the impact of the different clinical factors associated with survival on univariate analysis. The independent prognostic value of each significant factor was subsequently explored on multivariate analysis using a stepwise backward Cox proportional hazard model. Variables with a $P$-value $>0.10$ were removed from the model. Analysis was performed using SPSS software version 11.5 (SPSS inc., Chicago, IL, USA) and GraphPad PRISM (GraphPad Software inc., La Jolla, CA, USA).

\section{RESULTS}

Patient demographics. The baseline patient characteristics of the Hammersmith Hospital derivation cohort are shown in Table 1. The majority was male (84\%) with a PS of $0-1(74 \%)$ and had a radiological diagnosis of stage III/IV disease (53\%) with predominant epithelioid histology (84\%). Twenty-two patients (41\%) had received frontline platinum-based chemotherapy. Twentyeight $(52 \%)$ patients received palliative talc pleurodesis following biopsy and were subsequently treated with best supportive care only. At the time of analysis, 50 patients $(80 \%)$ had died and the median OS was 8.4 months (95\% CI: 3.5-13.4). When categorised according to the EPS; 50 patients $(80 \%)$ had good risk and 13 patients $(20 \%)$ were of poor risk.

Axl and Gas-6 expression: relationship with patients' characteristics and survival. Representative sections of MPM stained for each of the investigated biomarker are shown in Figure 1. In the case of Axl, we observed a consistent cytoplasmic and membranous expression pattern in all the examined specimens. Occasional Axl immunostaining of peritumoural endothelial cells was seen in Axlpositive samples (Figure 1H). Gas-6 expression was predominantly cytoplasmic in tumour cells, with rare nuclear positivity seen in a minority of specimens regardless of histotype. No other stromal cells displayed a convincingly specific positivity to Axl or Gas-6. In primary MPM samples, HS values for Axl ranged from 0 to 300 (median 150), and $74 \%$ were categorised as showing high expression (defined as $\mathrm{HS} \geqslant 150$ ). Epithelial tumours had significantly higher Axl expression levels (median HS 180 range 0-300) compared with non-epithelial mesotheliomas (median 0, range $0-300)(P=0.03)$. In a subgroup analysis Axl expression correlated inversely with Ki-67 proliferative index: median Axl HS values for low proliferating tumours was 180 (range 0-270) vs 80 $(0-180)$ of high proliferating tumours $(P=0.03)$ (Figure $2 \mathrm{~A})$. Gas6 expression ranged from 0 to 180 (median 0 , mean 45), and $50 \%$ were categorised as showing high expression (defined as HS $>0$ ). No correlation could be found between the expression of Axl and Gas-6 $(P=0.25)$. No statistically significant correlation with
Table 1. Patient characteristics

\begin{tabular}{l|l|l|}
\hline Baseline characteristic & $\mathbf{n = 6 3}(\%)$ & Median (range) \\
\hline Gender & \\
\hline Male & $53(84)$ & \\
Female & $10(16)$ &
\end{tabular}

Age in years

\begin{tabular}{|l|l|l|}
\hline$<65$ & $31(49)$ & $65(41-87)$ \\
\hline$\geqslant 65$ & $32(51)$ & \\
\hline
\end{tabular}

Asbestos exposure

Documented

Not documented

Unknown

27 (43)

20 (32)

$16(25)$

ECOG performance status

\begin{tabular}{|l|r|}
\hline 0 & $23(36)$ \\
1 & $24(38)$ \\
2 & $9(14)$ \\
3 & $4(6)$ \\
Missing & $3(6)$
\end{tabular}

IMIG stage

I-II

III-IV

Missing

27 (43)

31 (49)

5 (8)

Histotype

Epithelioid

Non-epithelioid

$53(84)$

$10(16)$

\section{EORTC prognostic score}

\begin{tabular}{|l|l|l|}
\hline $\begin{array}{l}\text { Good prognosis } \\
\text { Poor prognosis }\end{array}$ & $50(80)$ & \\
\hline Albumin, $\mathrm{gl}^{-1}$ & & \\
\hline$<35 \mathrm{gl}^{-1}$ & $22(63)$ & $33(15-42)$ \\
$\geqslant 35 \mathrm{gl}^{-1}$ & $38(37)$ & \\
\hline
\end{tabular}

Haemoglobin, $\mathrm{gl}^{-1}$

\begin{tabular}{|l|l|l|}
\hline$<12$ & $19(32)$ & $12.5(8.2-17)$ \\
$\geqslant 12$ & $41(68)$ & \\
\hline
\end{tabular}

White blood cell count, $\times 10^{3} \mathrm{I}^{-1}$

\begin{tabular}{|l|l|l|}
\hline$<8.3$ & $32(53)$ & $8.3(4.3-17)$ \\
$\geqslant 8.3$ & $28(47)$ & \\
\hline
\end{tabular}

Platelet count, $\times 10^{3} \mathrm{I}^{-1}$

\begin{tabular}{|l|l|l|}
\hline$<400$ & $33(55)$ & $379(160-614)$ \\
$\geqslant 400$ & $27(45)$ & \\
\hline
\end{tabular}

Gas-6 expression

\begin{tabular}{|l|l}
\hline Low & $33(53)$ \\
\hline
\end{tabular}

High

$30(47)$

\section{Axl expression}

\begin{tabular}{|l|l}
\hline Low & $16(26)$ \\
High & $47(74)$
\end{tabular}

Treatments

Pleurectomy/decortication

Systemic chemotherapy

\begin{tabular}{l|l} 
Best supportive care & $28(52)$
\end{tabular}

$22(41)$

Abbreviations: IMIG = International Mesothelioma Interest Group; ECOG = Eastern Cooperative Oncology Group; EORTC = European Organisation for Research and Treatment of Cancer.

Derivation cohort $(n=63)$. 


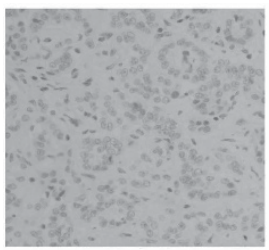

A

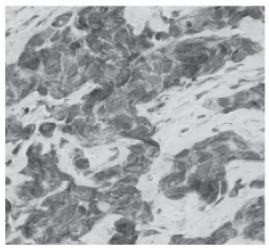

E

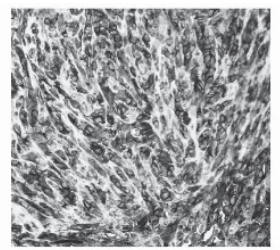

B

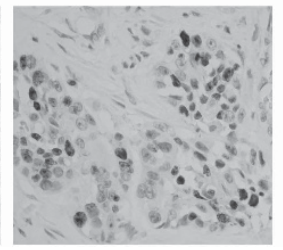

F

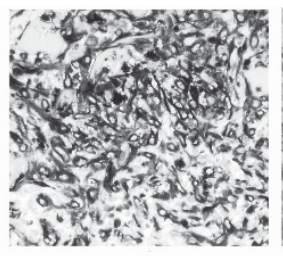

C

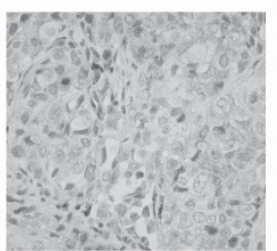

G

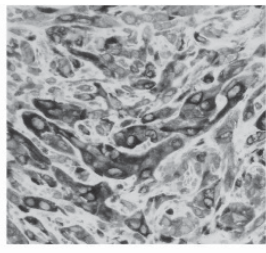

D

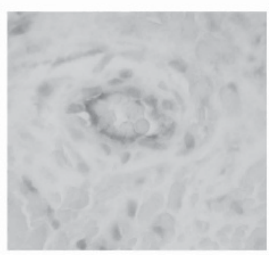

H

Figure 1. Expression of the candidate biomarkers in MPM by immunohistochemistry. Representative section of a case of MPM showing negative staining is seen in A. Axl expression in epithelial (B) and sarcomatoid MPM (C) appears with a typical diffused cytoplasmic and membranous pattern. A representative case showing strong cytoplasmic expression of Gas-6 is seen in D. Panel E is illustrative of a case with elevated VEGF-A expression, whereas in F an MPM sample with elevated expression of the Ki-67 nuclear antigen is shown. (G) Representative of a case with positive staining to hTERT. In $\mathrm{H}$ we show a high magnification micrograph of a peritumoural blood vessel with endothelial cells staining positively for Axl. Magnification $\times 400$ for $\mathbf{A}-\mathbf{G} ; \times 600$ for $\mathbf{H}$.

A
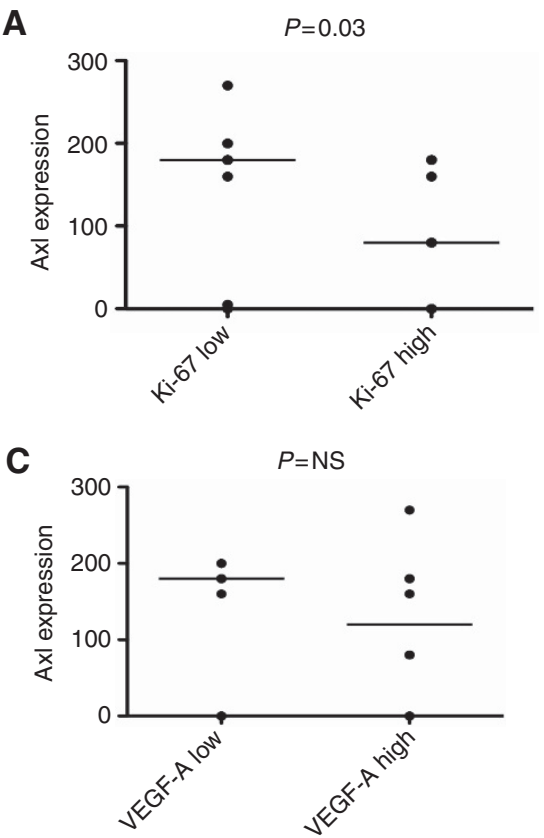

B

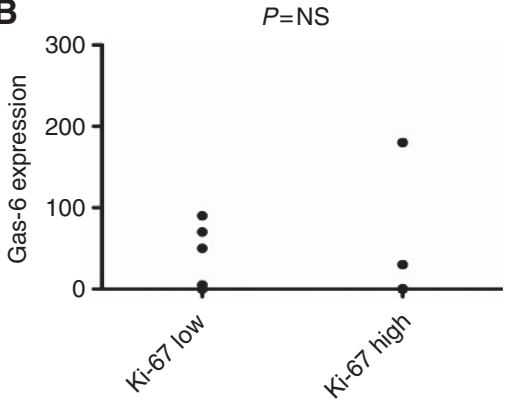

D

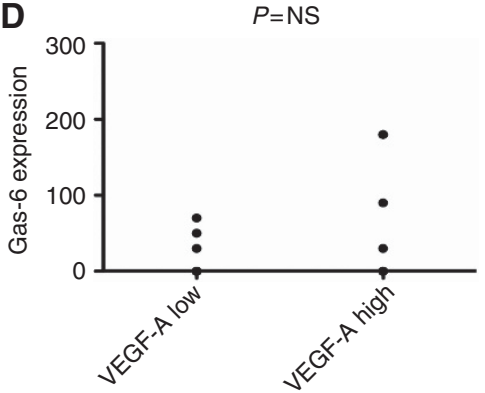

Figure 2. Aligned dot plot graphs showing the differential expression levels of Axl and Gas-6 according to Ki-67 immunopositivity (A, B) and VEGFA expression (C, D). Categorisation of Ki-67 and VEGF-A expression levels was carried out using the median values of the respective distributions as stated in the Methods section.

VEGF-A expression could be found for either Axl or Gas-6 (Figure 2B-D).

The prognostic value of Axl and Gas-6 overexpression was tested together with established prognostic factors in the derivation cohort. Univariate analyses revealed the following variables to predict for poor OS: low Axl expression $(P=0.001)$, IMIG stage $(P=0.05)$, PS $>1 \quad(P<0.001)$, albumin $<35 \mathrm{gl}^{-1} \quad(P<0.001)$, haemoglobin $<12 \mathrm{gl}^{-1} \quad(P<0.001)$. Multivariate analysis of survival confirmed reduced Axl expression $(P=0.01)$, PS $>1$ $(P=0.01)$, haemoglobin $<12 \mathrm{gl}^{-1}(P=0.04)$ and albumin $<35 \mathrm{gl}^{-1}(P<0.001)$ as independent predictors of worse OS (Table 2). Median OS for patients with Axl-negative tumours was 3.7 months (95\% CI 1.7-5.9), compared with 14.8 months (95\% CI 5.8-23.7) of the Axl-positive cases. (Figure $3 \mathrm{~A}$ and B). In order to further elucidate the mechanism by which Axl expression improves OS in patients with mesothelioma, we performed an ad hoc subgroup analysis on Axl-negative vs Axl overexpressing clinical specimens ( $n=10$ each group) to verify whether hTERT expression, a marker of unlimited proliferative potential, was influenced by Axl expression levels. We observed that mesothelioma samples positive for Axl were all negative for hTERT, and similarly 3 out of the 10 Axl negative samples chosen were positive for hTERT. However, the relationship between hTERT and Axl expression did not reach statistical significance $(P=0.06)$.

The prognostic role of Axl expression was reproduced in a validation set composed of 35 patients with similar characteristics: $62 \%$ male patients with median age 63 (range 43-92), predominantly epithelioid tumours (88\%) and median OS 9.9 months 
Table 2. Univariate and multivariate analysis of prognostic factors of overall survival (derivation cohort)

\begin{tabular}{|c|c|c|c|c|c|}
\hline \multirow[b]{3}{*}{ Variable } & \multirow[b]{3}{*}{$\mathbf{N}=63$} & \multicolumn{4}{|c|}{ Overall survival } \\
\hline & & \multicolumn{2}{|c|}{ Univariate analysis } & \multicolumn{2}{|c|}{ Multivariate analysis } \\
\hline & & Hazard ratio $(95 \% \mathrm{Cl})$ & $P$-value & Hazard ratio $(95 \% \mathrm{Cl})$ & $\boldsymbol{P}$-value \\
\hline \multicolumn{6}{|l|}{ Imig stage } \\
\hline I-II/III-IV & $27 / 31$ & $1.4(1.0-2.0)$ & $0.05^{*}$ & & \\
\hline \multicolumn{6}{|l|}{ ECOG PS } \\
\hline $0-1 / \geqslant 2$ & $47 / 13$ & $4.5(2.2-9.1)$ & $<0.001^{*}$ & $3.0(1.3-7.0)$ & $0.01^{*}$ \\
\hline \multicolumn{6}{|l|}{ Histotype } \\
\hline Epitelioid/non-epithelioid & $53 / 10$ & $1.2(0.5-2.6)$ & 0.70 & & \\
\hline \multicolumn{6}{|l|}{ Albumin, $\mathrm{gl}^{-1}$} \\
\hline$\geqslant 35 /<35 \mathrm{gl}^{-1}$ & $38 / 22$ & $6.4(3.1-16.5)$ & $<0.001^{*}$ & $5.0(2.3-11.5)$ & $<0.001^{*}$ \\
\hline \multicolumn{6}{|l|}{ Haemoglobin } \\
\hline$\geqslant 12 /<12$ & $41 / 19$ & $4.0(2.1-7.9)$ & $<0.001^{*}$ & $2.1(1.0-4.3)$ & $0.04^{*}$ \\
\hline \multicolumn{6}{|l|}{ EPS } \\
\hline Good/poor prognosis & $50 / 13$ & $1.4(0.7-2.8)$ & 0.37 & & \\
\hline \multicolumn{6}{|l|}{ Axl expression } \\
\hline Negative/positive & $16 / 47$ & $0.3(0.2-0.6)$ & $0.001^{*}$ & $0.3(0.2-0.8)$ & $0.01^{*}$ \\
\hline \multicolumn{6}{|l|}{ Gas-6 expression } \\
\hline Negative/positive & $33 / 30$ & $0.7(0.4-1.4)$ & 0.35 & & \\
\hline
\end{tabular}
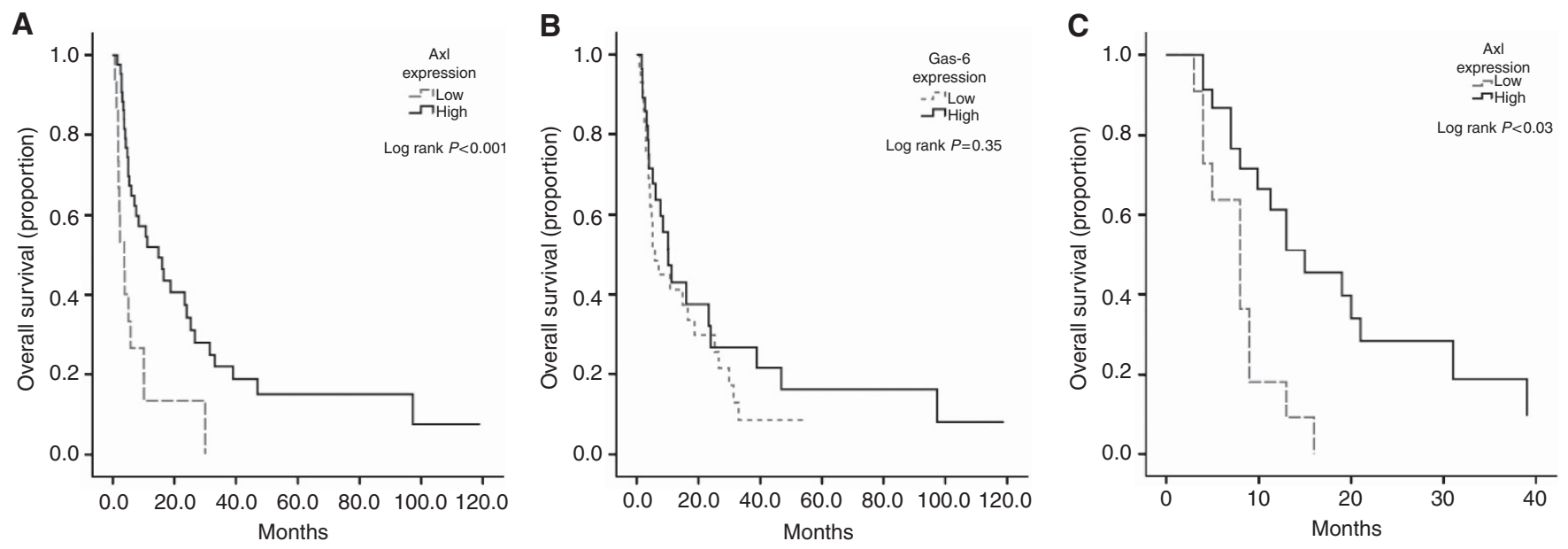

Figure 3. Kaplan-Meier survival curves for patients with MPM included in the derivation cohort stratified by Axl (A) and Gas-6 expression (B). (C) shows the survival probability of patients included in the validation set, stratified by Axl expression.

(95\% CI 6.0-14.0) (Table 3). In the validation set, patients with elevated Axl expression $(n=24,68 \%)$ had a significantly better OS (15.0 months, 95\% CI 7.0-23.0) than patients with Axl-negative tumours (8.0 months, 95\% CI 4.9-11.1, hazard ratio for OS 0.3 , $95 \%$ CI $0.2-0.7, P=0.003$ ) (Figure 3C). The lack of prognostic role for Gas-6 expression was confirmed in the validation set $(P=0.78)$.

\section{DISCUSSION}

Dysregulation of membrane-bound RTKs and their downstream signalling pathways is a common event in pleural carcinogenesis, where a significant upregulation of multiple RTKs including 


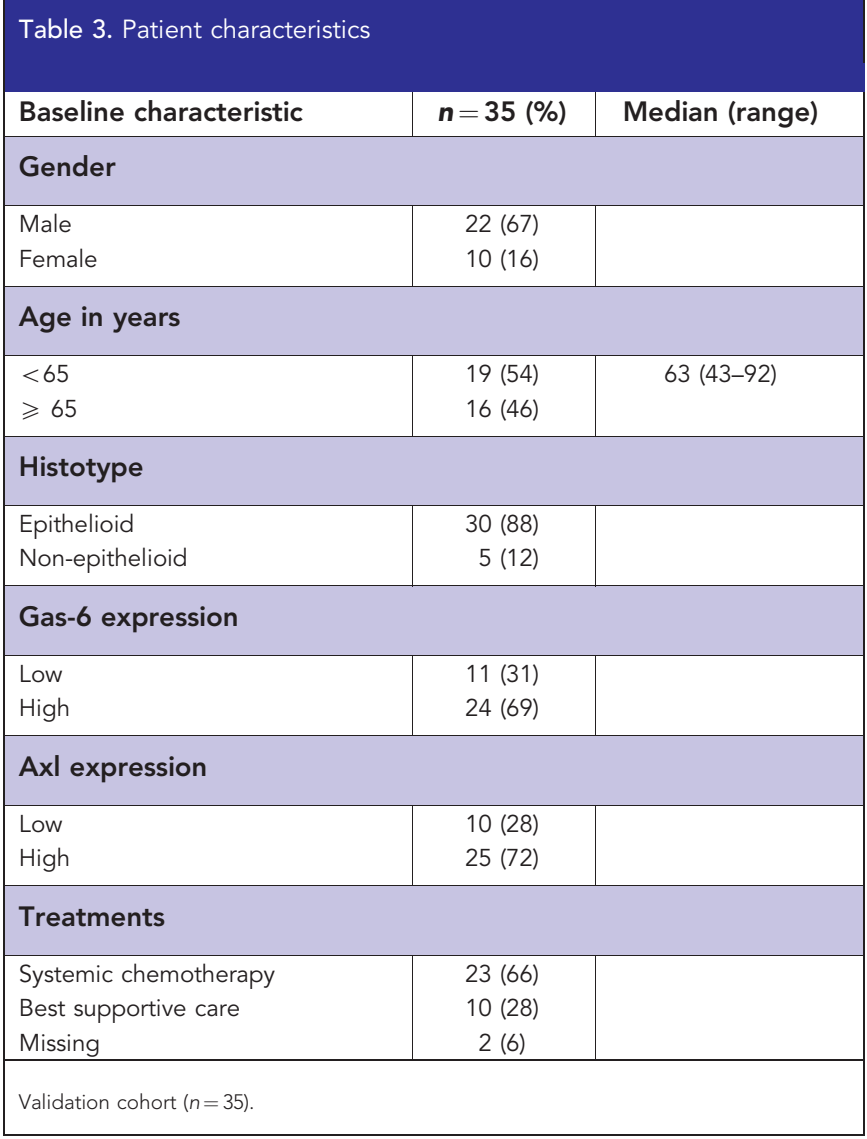

EGF-R, c-MET, c-ERB-b3 and Axl is seen in neoplastic cells compared with their normal mesothelial counterparts ( $\mathrm{Ou}$ et al, 2011b).

As the aberrant activation of RTKs is crucial to maintain tumour cell proliferation and facilitate evasion from apoptosis, a considerable interest has emerged for RTKs both as tissue biomarkers to predict the biologic aggressiveness of the disease as well as putative therapeutic targets, in a context where the survival benefit that can be offered by currently available treatments is notoriously limited.

Axl has been shown to modulate several aspects of cancer progression through simple overexpression rather than by activating mutations (Burchert et al, 1998), being involved in the acquisition of cell motility and invasive potential through the upregulation of Snail, vimentin and EMT transcriptional programs (Vuoriluoto et al, 2011). Strong evidence supports the role of Axl/ Gas-6 pathway in promoting endothelial cell proliferation and neoangiogenesis (Holland et al, 2005), and the secretion of Gas-6 by peritumoural stromal cells is part of a recognised biochemical crosstalk that acts on Axl-positive tumour cell clones exerting mitogenic effects (Loges et al, 2010).

The qualification of $\mathrm{Axl}$ as a transforming oncogene regulating the proliferation and invasiveness of MPM relies on preclinical studies, where the inhibition of Axl using selective tyrosine kinase inhibitors determined significant antiproliferative effects in vitro (Ou et al, 2011a).

In the present study, the first to determine the prevalence and clinicopathological significance of Axl and Gas-6 expression in primary MPM clinical samples, we showed that Axl overexpression is a molecular trait characterising $\sim 75 \%$ of MPM, while immunohistochemical detection of Gas-6 is common in at least half of the cases we tested.

In addition, we identified a significant and independent prognostic role for the oncogene Axl in MPM. Unlike other tumours, where Axl overexpression negatively impacts on patients' prognosis (Hutterer et al, 2008; Gjerdrum et al, 2010; Song et al, 2011), we found that Axl expression exerts a protective effect on patients' survival independent from other validated prognostic factors such as tumour stage, PS, low haemoglobin and hypoalbuminaemia (Kao et al, 2012). To ascertain that this finding was not due to patient selection bias or to the relatively small sample size of our derivation cohort, we opted to validate our observation using an independent data set of patients with similar characteristics obtained from another institution. Strikingly, the results of our survival analysis were reproduced in full, suggesting that our observation is generally representative of MPM.

This is not the first paper to describe the protective role of overexpression of oncogenes on patients' survival.

In the case of EGF-R, for instance, whose wild-type form is overexpressed in up to 50\% of MPM (Destro et al, 2006), the higher expression rates displayed by epithelial tumours, typically characterised by a relatively less aggressive course, can at least in part explain the better survival outcomes seen in patients with high EGF-R immunopositivity (Edwards et al, 2006). Despite initial evidence suggesting that $\mathrm{Axl}$ is preferentially upregulated in sarcomatoid MPM (Ou et al, 2011a), our study, in line with others investigating EMT markers such as c-MET (Levallet et al, 2012), in fact shows a relative predominance of Axl expression in epithelioid tumours, a finding that may not only justify the protective effect of Axl expression on patients' survival we have seen but also confirm that the EMT phenotype is not only restricted to sarcomatoid mesotheliomas as previously thought.

In the specimens we analysed, MPM often acted as a primary source of Gas-6, suggesting the presence of autocrine stimulatory loops within the tumour itself (Gjerdrum et al, 2010). However, Gas-6 expression levels did not correlate with those of Axl in our study, suggesting that the activation of Axl-mediated signalling may also happen independently from the presence of Gas-6, for which no definite prognostic role could be inferred from our data. It can be further hypothesised that Axl overexpression as a result of copy number gain has in fact been shown to be sufficient to exert oncogenic properties even in absence of its ligand (Johnson et al, 2012).

To obtain further insight into the biologic relevance of Axl and Gas-6 in MPM, we correlated their expression with Ki-67 and VEGF-A, two validated tissue biomarkers reflecting the proliferative and angiogenic potential of tumour cells. As shown in Figure 2 we did not find a significant relationship between Axl/Gas-6 expression and VEGF-A immunopositivity. However, we observed that median Axl expression levels were significantly lower in highly proliferating MPM (i.e. Ki-67 $\geqslant 30 \%$ ). The inverse relationship between Axl expression and proliferation prompted us to consider whether Axl overexpression could trigger cellular senescence, in keeping with previously published evidence showing that at the highest intensity of their activation, certain oncogenes such as Ras (Sarkisian et al, 2007) or phosphatidil-inositol-3 kinase (PI3K)/AKT (Majumder et al, 2008) may determine paradoxical antiproliferative responses globally termed as oncogene-induced senescence (Mallette and Ferbeyre, 2007). To test this hypothesis, that we thought relevant as Axl is a renowned activator of the PI3K/AKT pathway (Ruan and Kazlauskas, 2012) and previous report showed that higher levels phospho-AKT and its downstream transcription factor Foxo3a conferred better prognosis in MPM (Cedres et al, 2012), we performed an ad hoc subgroup analysis on Axl-negative vs Axl overexpressing clinical specimens ( $n=10$ each group) to verify whether hTERT expression, a marker of unlimited proliferative potential, was influenced by Axl expression levels. We observed that hTERT expression was restricted to 3/10 Axl-negative tumours, whereas none of the Axl-positive samples we tested were found to express hTERT. Such finding may suggest a potential role for Axl overexpression in 
promoting cellular senescence, which may at least in part account for the protective role of Axl in influencing patient's prognosis. However, as the relationship between the two markers did not reach statistical significance in our study, our conclusions should be interpreted as preliminary and the interplay between Axl expression and cellular senescence should be further explored in mechanistic studies.

In aggregate, our results validate for the first time the expression of Axl and Gas-6 in a multi-institutional series of MPM clinical specimens, confirming an independent prognostic value for Axl overexpression whose robustness relies on a process of external validation. The predictive power of Axl expression in estimating patients' survival appears stronger than that of commonly used prognostic factors including tumour stage and EORTC prognostic score. Not surprisingly, the latter was not predictive of patients' survival in our cohort, in line with previous reports refuting its independent prognostic power (Kao et al, 2010; Pinato et al, 2012). We interpret the lack of prognostic power observed for histotype classification as a consequence of the overrepresentation of epithelioid cases, amounting to $>80 \%$ of the total.

Despite the relatively small sample size, the number of deaths on which our survival analysis was powered and the process of external validation we utilised guarantees for the reliability of our statistical analysis.

To conclude, we found that the immunohistochemical expression of Axl in primary MPM cells accurately predicted for longer OS in two independent cohorts of patients. Immunohistochemical evaluation of Axl expression status in diagnostic biopsy specimens may therefore improve prognostic prediction of MPM in routine clinical practice.

\section{ACKNOWLEDGEMENTS}

We thank Professor Stefano Ferrero-Bogetto (University of Milan, Italy) for having supported this study. We would like to thank Professor Gian Carlo Avanzi, whose research into the biologic relevance of Gas-6 and Axl in cancer physiology have inspired this work.

\section{AUTHOR CONTRIBUTIONS}

All authors have been contributing to: (1) study conception and design, or analysis and interpretation of the data (2) drafting or revision of the manuscript. All authors have approved the final version to be published.

\section{REFERENCES}

Arber DA, Weiss LM, West RB (2004) CD117 expression in mesothelioma. Mod Pathol 17(8): 1021.

Burchert A, Attar EC, McCloskey P, Fridell YW, Liu ET (1998) Determinants for transformation induced by the Axl receptor tyrosine kinase. Oncogene 16(24): 3177-3187.

Cedres S, Montero MA, Martinez P, Martinez A, Rodriguez-Freixinos V, Torrejon D, Gabaldon A, Salcedo M, Ramon YCS, Felip E (2012) Exploratory analysis of activation of PTEN-PI3K pathway and downstream proteins in malignant pleural mesothelioma (MPM). Lung Cancer 77(1): 192-198.

Destro A, Ceresoli GL, Falleni M, Zucali PA, Morenghi E, Bianchi P, Pellegrini C, Cordani N, Vaira V, Alloisio M, Rizzi A, Bosari S, Roncalli M (2006) EGFR overexpression in malignant pleural mesothelioma. An immunohistochemical and molecular study with clinico-pathological correlations. Lung Cancer 51(2): 207-215.

Edwards JG, Swinson DE, Jones JL, Waller DA, O’Byrne KJ (2006) EGFR expression: associations with outcome and clinicopathological variables in malignant pleural mesothelioma. Lung Cancer 54(3): 399-407.
Fennell DA, Parmar A, Shamash J, Evans MT, Sheaff MT, Sylvester R, Dhaliwal K, Gower N, Steele J, Rudd R (2005) Statistical validation of the EORTC prognostic model for malignant pleural mesothelioma based on three consecutive phase II trials. J Clin Oncol 23(1): 184-189.

Fridell YW, Jin Y, Quilliam LA, Burchert A, McCloskey P, Spizz G, Varnum B, Der C, Liu ET (1996) Differential activation of the Ras/extracellularsignal-regulated protein kinase pathway is responsible for the biological consequences induced by the Axl receptor tyrosine kinase. Mol Cell Biol 16(1): 135-145.

Garland LL, Rankin C, Gandara DR, Rivkin SE, Scott KM, Nagle RB, KleinSzanto AJ, Testa JR, Altomare DA, Borden EC (2007) Phase II study of erlotinib in patients with malignant pleural mesothelioma: a Southwest Oncology Group Study. J Clin Oncol 25(17): 2406-2413.

Gjerdrum C, Tiron C, Hoiby T, Stefansson I, Haugen H, Sandal T, Collett K, Li S, McCormack E, Gjertsen BT, Micklem DR, Akslen LA, Glackin C, Lorens JB (2010) Axl is an essential epithelial-to-mesenchymal transitioninduced regulator of breast cancer metastasis and patient survival. Proc Natl Acad Sci USA 107(3): 1124-1129.

Greillier L, Baas P, Welch JJ, Hasan B, Passioukov A (2008) Biomarkers for malignant pleural mesothelioma: current status. Mol Diagn Ther 12(6): 375-390.

Hasanbasic I, Cuerquis J, Varnum B, Blostein MD (2004) Intracellular signaling pathways involved in Gas6-Axl-mediated survival of endothelial cells. Am J Physiol Heart Circ Physiol 287(3): H1207-H1213.

Hasani A, Alvarez JM, Wyatt JM, Bydder S, Millward M, Byrne M, Musk AW, Nowak AK (2009) Outcome for patients with malignant pleural mesothelioma referred for Trimodality therapy in Western Australia J Thorac Oncol 4(8): 1010-1016.

Holland SJ, Powell MJ, Franci C, Chan EW, Friera AM, Atchison RE, McLaughlin J, Swift SE, Pali ES, Yam G, Wong S, Lasaga J, Shen MR, Yu S, Xu W, Hitoshi Y, Bogenberger J, Nor JE, Payan DG, Lorens JB (2005) Multiple roles for the receptor tyrosine kinase axl in tumor formation. Cancer Res 65(20): 9294-9303.

Hutterer M, Knyazev P, Abate A, Reschke M, Maier H, Stefanova N, Knyazeva T, Barbieri V, Reindl M, Muigg A, Kostron H, Stockhammer G, Ullrich A (2008) Axl and growth arrest-specific gene 6 are frequently overexpressed in human gliomas and predict poor prognosis in patients with glioblastoma multiforme. Clin Cancer Res 14(1): 130-138.

Jean D, Daubriac J, Le Pimpec-Barthes F, Galateau-Salle F, Jaurand MC (2012) Molecular changes in mesothelioma with an impact on prognosis and treatment. Arch Pathol Lab Med 136(3): 277-293.

Johnson CE, Gorringe KL, Thompson ER, Opeskin K, Boyle SE, Wang Y, Hill P, Mann GB, Campbell IG (2012) Identification of copy number alterations associated with the progression of DCIS to invasive ductal carcinoma. Breast Cancer Res Treat 133(3): 889-898.

Joshi TK, Gupta RK (2004) Asbestos in developing countries: magnitude of risk and its practical implications. Int J Occup Med Environ Health 17(1): 179-185.

Kao SC, Pavlakis N, Harvie R, Vardy JL, Boyer MJ, van Zandwijk N, Clarke SJ (2010) High blood neutrophil-to-lymphocyte ratio is an indicator of poor prognosis in malignant mesothelioma patients undergoing systemic therapy. Clin Cancer Res 16(23): 5805-5813.

Kao SC, Vardy J, Chatfield M, Corte P, Pavlakis N, Clarke C, van Zandwijk N, Clarke S (2012) Validation of prognostic factors in malignant pleural mesothelioma: a retrospective analysis of data from patients seeking compensation from the New South Wales Dust Diseases Board. Clin Lung Cancer 14(1): 70-77.

Levallet G, Vaisse-Lesteven M, Le Stang N, Ilg AG, Brochard P, Astoul P, Pairon JC, Bergot E, Zalcman G, Galateau-Salle F (2012) Plasma cell membrane localization of c-MET predicts longer survival in patients with malignant mesothelioma: a series of 157 cases from the MESOPATH Group. J Thorac Oncol 7(3): 599-606.

Loges S, Schmidt T, Tjwa M, van Geyte K, Lievens D, Lutgens E, Vanhoutte D, Borgel D, Plaisance S, Hoylaerts M, Luttun A, Dewerchin M, Jonckx B, Carmeliet P (2010) Malignant cells fuel tumor growth by educating infiltrating leukocytes to produce the mitogen Gas6. Blood 115(11): 2264-2273.

Majumder PK, Grisanzio C, O’Connell F, Barry M, Brito JM, Xu Q, Guney I, Berger R, Herman P, Bikoff R, Fedele G, Baek WK, Wang S, Ellwood-Yen K, Wu H, Sawyers CL, Signoretti S, Hahn WC, Loda M, Sellers WR (2008) A prostatic intraepithelial neoplasia-dependent p27 Kip1 checkpoint induces senescence and inhibits cell proliferation and cancer progression. Cancer Cell 14(2): 146-155. 
Mallette FA, Ferbeyre G (2007) The DNA damage signaling pathway connects oncogenic stress to cellular senescence. Cell Cycle 6(15): 1831-1836.

Mathy A, Baas P, Dalesio O, van Zandwijk N (2005) Limited efficacy of imatinib mesylate in malignant mesothelioma: a phase II trial. Lung Cancer 50(1): 83-86.

Ou WB, Corson JM, Flynn DL, Lu WP, Wise SC, Bueno R, Sugarbaker DJ, Fletcher JA (2011a) AXL regulates mesothelioma proliferation and invasiveness. Oncogene 30(14): 1643-1652.

Ou WB, Hubert C, Corson JM, Bueno R, Flynn DL, Sugarbaker DJ, Fletcher JA (2011b) Targeted inhibition of multiple receptor tyrosine kinases in mesothelioma. Neoplasia 13(1): 12-22.

Pinato DJ, Mauri FA, Ramakrishnan R, Wahab L, Lloyd T, Sharma R (2012) Inflammation-based prognostic indices in malignant pleural mesothelioma. J Thorac Oncol 7(3): 587-594.

Rena O, Casadio C (2012) Extrapleural pneumonectomy for early stage malignant pleural mesothelioma: a harmful procedure. Lung Cancer 77(1): 151-155.

Ruan GX, Kazlauskas A (2012) Axl is essential for VEGF-A-dependent activation of PI3K/Akt. EMBO J 31(7): 1692-1703.

Rusch VW (1995) A proposed new international TNM staging system for malignant pleural mesothelioma. From the International Mesothelioma Interest Group. Chest 108(4): 1122-1128.

Sarkisian CJ, Keister BA, Stairs DB, Boxer RB, Moody SE, Chodosh LA (2007) Dose-dependent oncogene-induced senescence in vivo and its evasion during mammary tumorigenesis. Nat Cell Biol 9(5): 493-505.

Shi SR, Key ME, Kalra KL (1991) Antigen retrieval in formalin-fixed, paraffinembedded tissues: an enhancement method for immunohistochemical staining based on microwave oven heating of tissue sections. J Histochem Cytochem 39(6): 741-748.

Song X, Wang H, Logsdon CD, Rashid A, Fleming JB, Abbruzzese JL, Gomez HF, Evans DB (2011) Overexpression of receptor tyrosine kinase Axl promotes tumor cell invasion and survival in pancreatic ductal adenocarcinoma. Cancer 117(4): 734-743.

Treasure T, Lang-Lazdunski L, Waller D, Bliss JM, Tan C, Entwisle J, Snee M, O’Brien M, Thomas G, Senan S, O'Byrne K, Kilburn LS, Spicer J, Landau D, Edwards J, Coombes G, Darlison L, Peto J (2011) Extra-pleural pneumonectomy versus no extra-pleural pneumonectomy for patients with malignant pleural mesothelioma: clinical outcomes of the Mesothelioma and Radical Surgery (MARS) randomised feasibility study. Lancet Oncol 12(8): 763-772.

Vogelzang NJ, Rusthoven JJ, Symanowski J, Denham C, Kaukel E, Ruffie P, Gatzemeier U, Boyer M, Emri S, Manegold C, Niyikiza C, Paoletti P (2003) Phase III study of pemetrexed in combination with cisplatin versus cisplatin alone in patients with malignant pleural mesothelioma. J Clin Oncol 21(14): 2636-2644.

Vuoriluoto K, Haugen H, Kiviluoto S, Mpindi JP, Nevo J, Gjerdrum C, Tiron C, Lorens JB, Ivaska J (2011) Vimentin regulates EMT induction by Slug and oncogenic $\mathrm{H}$-Ras and migration by governing Axl expression in breast cancer. Oncogene 30(12): 1436-1448.

Weder W, Stahel RA, Bernhard J, Bodis S, Vogt P, Ballabeni P, Lardinois D, Betticher D, Schmid R, Stupp R, Ris HB, Jermann M, Mingrone W, Roth AD, Spiliopoulos A (2007) Multicenter trial of neo-adjuvant chemotherapy followed by extrapleural pneumonectomy in malignant pleural mesothelioma. Ann Oncol 18(7): 1196-1202.

This work is published under the standard license to publish agreement. After 12 months the work will become freely available and the license terms will switch to a Creative Commons AttributionNonCommercial-Share Alike 3.0 Unported License. 